\section{The Design of Multi-Lead-Compensators for Stabilization and Pole Placement in Double-Integrator Networks}

\author{
Yan Wan, Member, IEEE, Sandip Roy, Member, IEEE, \\ Ali Saberi, Fellow, IEEE, and \\ Anton Stoorvogel, Senior Member, IEEE
}

\begin{abstract}
We study decentralized controller design for stabilization and pole-placement, in a network of autonomous agents with double-integrator internal dynamics and arbitrary observation topology. We show that a simple multi-lead-compensator architecture, in particular one in which each agent uses a derivative-approximation compensator with three memory elements, can achieve both stabilization and effective pole placement while subdividing complexity/actuation among the agents. Through a scaling argument, we also demonstrate that the multi-lead-compensator can stabilize the double-integrator network under actuator saturation constraints.
\end{abstract}

Index Terms-Decentralized control, lead compensator, pole placement, saturation, stabilization.

\section{INTRODUCTION}

Through our studies of controller design in several modern dynamical networks [1]-[6], we have become convinced that network structure (i.e., the sensing/interaction interconnection structure among the network's components or agents) is critical in driving network dynamics, and hence must be exploited in controller design. Due to the crucial role played by the network structure, novel decentralized controller architectures that have the following two features are badly needed in dynamical network control applications: 1) control complexity and actuation are roughly equally contributed by all the agents, and 2) the controller can address control/algorithmic tasks in networks with general sensing and/or interaction topologies and constraints such as actuator saturation. In this work, we introduce a novel decentralized control scheme for shaping the dynamics of networks with arbitrary sensing structures, which we call the multi-lead-compensator. We show that the multi-lead-compensator-precisely, an linear time-invariant (LTI) decentralized state-space controller with a small number of memory elements used in each channel, that approximates a multiple-derivative feedback - can be designed to achieve stabilization and pole-placement in a simple autonomous-agent network model with a general sensing structure. We also adapt the design for stabilization under actuator saturation.

Our efforts are deeply connected with two bodies of research: 1) recent efforts on autonomous-agent network control, and 2) studies of decentralized control. The many recent works on autonomous-agent

Manuscript received September 21, 2008; revised May 25, 2009; accepted August 24, 2010. Date of publication September 02, 2010; date of current version December 02, 2010. The work was supported by National Science Foundation Grants ECS-0528882 and ECCS-0725589, NAVY Grants ONR KKK777SB001 and ONR KKK60SB0012, and National Aeronautics and Space Administration Grant NNA06CN26A. Recommended by Associate Editor R. D. Braatz.

Y. Wan is with the Department of Electrical Engineering, University of North Texas, Denton, TX, 76201 USA (e-mail: yan.wan@unt.edu).

S. Roy and A. Saberi are with the Department of Electrical Engineering, Washington State University, Pullman, WA 99164-2752 USA (e-mail: sroy@eecs.wsu.edu; saberi@eecs.wsu.edu).

A. Stoorvogel is with the Department of Electrical Engineering, Mathematics and Computer Science, University of Twente, Enschede 7500 AE, The Netherlands (e-mail: a.a.stoorvogel@utwente.nl).

Digital Object Identifier 10.1109/TAC.2010.2073010 network control are fundamentally derived from prominent work by Chua and his colleagues [7], [8] on network synchronization (see also e.g., [9]). Chua gave necessary and sufficient conditions for a diffusive network with identical agents to achieve synchronization [7], and in turn developed a graphical interpretation of the condition [8]. Fax and Murray in [10] and Pogromsky in [11] brought forth control interpretations to the synchronization tasks in a diffusive network (in the case where identical controllers are used at each channel), and thus gave conditions for synchronization through control in this case. More recently, numerous other dynamical network tasks such as formation, agreement, and alignment [6], [12]-[15] have been addressed in essentially similar ways.

Development of stabilizing network controllers has also been pursued under the heading of decentralized control. In a seminal work [16], Wang and Davison give an implicit sufficient and necessary condition for the existence of a stabilizing time-invariant dynamic controller for a general decentralized system, but their general result does not yield a practical controller design. Meanwhile, many efforts (see [17]) view the network interconnections as disturbances, and provide controller designs based on that assumption. In an alternate direction, building on [16], several works have pursued decentralized stabilization and pole-assignment through static pre-feedback to make the system controllable and observable from one channel followed by (centralized) dynamical controller design at this channel [18], [19]. Despite these efforts, practical decentralized controller design [4] remains difficult for complex interaction/sensing structures.

Now let us emphasize the contribution of the multi-lead-compensator design to these research directions. Our aim here is to obtain a systematic construction of a decentralized controller for not only stabilization but pole placement for a double-integrator network [6] that has several properties: 1) it is able to exploit the network topology and and heterogeneity in the control architecture to achieve stability/performance for general sensing structures; 2) it distributes complexity and actuation effort appropriately among the agents in the network; 3) it can be adapted to achieve stabilization under practical constraints such as actuator saturation. Although our focus here is on double-integrator networks, we note that the methodology holds promise for more general plants (see [20], [21]).

Let us also discuss numerous recent efforts on constructing network controllers or topologies for high performance, that are aligned with our efforts here. Of interest, Boyd and his coworkers have used linear matrix inequality (LMI) techniques to optimize a network's settling dynamics, through design of an associated graph [22]. In complement, building on a classical result of Fisher and Fuller [23], we have taken a structural approach to performance optimization through graph-edge and static decentralized controller design [2]. [24]-[26]. This meshed control-theory and algebraic-graph-theory strategy has yielded designs for several families of network-interaction models and performance criteria, and has also permitted us to address the partial design problem. Meanwhile, in [4], we introduced the multiplederivative-feedback- based paradigm for decentralized controller design, but considered delay-feedback implementations. Our efforts in this short note enhance these designs through use of simple dynamical controllers, which can give significant freedom in shaping the network's response (for instance, allowing pole placement).

Finally, our study is deeply connected to efforts to control networks with actuator saturation. Of interest, Stoorvogel and coworkers have given sufficient conditions on linear systems for existence of decentralized controllers under saturation, in particular showing that stabilization is possible when the open-loop decentralized plant has 1) only closed-left-half-plane eigenvalues, 2) decentralized fixed modes only 
in the open left-half-plane, and 3) $j w$-axis axis eigenvalues with algebraic multiplicity 1 [30]. However, this study does not address the case where $j w$-axis eigenvalues are repeated, nor does it give a practical controller design. Meanwhile, a couple of recent works have given sufficient conditions for stabilizing a double-integrator network under actuator saturation [6], [15], but these apply only to some special network topologies.

The remainder of the article is organized as follows. In Section II, we formulate the double-integrator network model. Section III addresses design of controllers for stabilization and pole-placement in the doubleintegrator network model, while Section IV addresses the case with actuator saturation.

\section{PROBLEM Formulation}

Let us introduce the double-integrator network, i.e., a decentralized system consisting of $n$ autonomous agents with double-integrator internal dynamics whose (scalar) observations are linear combinations of multiple agents' states. Precisely, we assume that each agent $i$ has internal dynamics $\ddot{x}_{i}=u_{i}$, where we refer to $x_{i}$ as the position state of agent $i$ and $\dot{x}_{i}$ as the velocity state, and $u_{i}$ is the input to agent $i$. For notational convenience, we define the full position state as $\mathbf{x}=$ $\left[\begin{array}{lll}x_{1} & \ldots & x_{n}\end{array}\right]^{T}$ and the full input as $\mathbf{u}=\left[\begin{array}{lll}u_{1} & \ldots & u_{n}\end{array}\right]^{T}$. We assume each agent $i$ makes a scalar observation $y_{i}=\mathbf{g}_{i}^{T} \mathbf{x}$, i.e., that its observation is a linear combination of the position states of various agents. We find it convenient to stack the observations into a vector, i.e., $\mathbf{y}=\left[\begin{array}{lll}y_{1} & \ldots & y_{n}\end{array}\right]^{T}$. We also stack vectors $\mathbf{g}_{i}^{T}$ to form a topology matrix $G=\left[\begin{array}{lll}\mathbf{g}_{1} & \cdots & \mathbf{g}_{n}\end{array}\right]^{T}$, that captures the sensing/communication among the agents. In short, a double-integrator network comprises $n$ agents that together have the dynamics

$$
\ddot{\mathbf{x}}=\mathbf{u}, \mathbf{y}=G \mathbf{x}
$$

where each agent $i$ makes an observation $y_{i}$ and can set the input $u_{i}$. We also consider a double-integrator network with agents that are subject to actuator saturation. That is, we consider a network of $n$ agents that have the dynamics

$$
\ddot{\mathbf{x}}=\sigma(\mathbf{u}), \mathbf{y}=G \mathbf{x}
$$

where $\sigma()$ represents a standard saturation function applied elementwise. Let us call this model the double-integrator network with saturation.

Our goal is to design a linear decentralized controller (mappings from each $y_{i}$ to $u_{i}$ ) to stabilize the double-integrator-network's dynamics. As a further step, we seek a pole-placement controller, i.e., one that achieves the classical controller design goal of placing the eigenvalues of the closed-loop dynamics at desirable locations. This decentralized controller design problem for the double-integrator network has wide application, see e.g., [6].

\section{Stabilizationand Pole-Placement For THE DOUBLE-INTEGRATOR NETWORK}

For the double-integrator network, it is necessary and sufficient for stabilization that $G$ has full rank [6], regardless of whether centralized or decentralized control is considered and regardless of whether a linear or a nonlinear time-varying (NLTV) controller is used (see also [16], [27]). Here, we will demonstrate not only stabilization but effective pole placement for arbitrary full rank $G$ using the most limited of these schemes, namely an LTI state-space decentralized controller. In fact, we will show that a very simple controller-one that has third-order dynamics at each channel-suffices.

Our multi-lead-compensator design is based on 1) construction of a high-gain feedback of multiple output derivatives up to degree 2 at each agent, to place the close-loop poles in desired locations in the open left half plane (OLHP); and 2) approximation of the multiplederivative controller with lead compensators. The philosophy of our design is that, for double-integrator networks, high gain feedback of output derivatives up to the degree of 2 can permit each agent to recover its local state information [4], and hence permit pole placement and stabilization. We emphasize that the novelty of the design lies in the unusual use of one higher derivative (here, the second derivative, or in other words the derivative equal to the relative degree of the local plant) in feedback. This is in contrast to the centralized setting, where controllers (whether designed using the observer-plus-state-feedback paradigm or in other ways) at their essence feed back derivatives up to one less than the plant's relative degree to achieve stabilization and pole placement [28]. We refer the reader to our concurrent works [4], [20] for discussion regarding the much broader application of the multiplederivative-feedback architecture in decentralized control.

Since derivative controllers cannot directly be used due to their unbounded high frequency gains, we pursue implementation using a leadcompensator scheme. We stress that, in that a derivative equal to the relative degree of the plant is being used in feedback, the implementation here through lead compensation is not routine (see e.g., the work on neutral-type delay-differential equations, such as [29]); nevertheless, we have been able to obtain a working control scheme for arbitrary full rank $G$. Specifically, the lead compensation scheme produces poles close to those of the derivative controller and also extra poles far inside the OLHP, and hence achieves stabilization and high performance.

The dynamical controller design presented here relies on Fisher and Fuller's classical result [23]. Let us describe Fisher and Fuller's result, before introducing and proving our main theorem.

Theorem 1: (Fisher and Fuller) Consider an $n \times n$ matrix $A$. If the matrix $A$ has a nested sequence of $n$ principal minors that all have full rank, then there exists a diagonal matrix $K$ such that the eigenvalues of $K A$ are in the open left half plane, and in fact on the negative real axis.

We note that the primary goal of Fisher and Fuller in their work was to demonstrate that the eigenvalues can be placed in a half-plane, but their construction of a diagonal scaling (1) yields real eigenvalues.

The following theorem, our main result, formalizes that stabilization and pole-placement can be achieved generally in the double-integrator network using third-order compensators at each channel. The proof of the theorem makes explicit the compensator design. Specifically, we describe how to design a controller so that sets of $n$ closed-loop eigenvalues can be placed arbitrarily near to two desired locations (closed under conjugation) in the complex plane, while the remaining $3 n$ eigenvalues are placed arbitrarily far left in the complex plane. Here is a formal statement:

Theorem 2: Consider a double-integrator network with arbitrary invertible topology matrix $G$. Proper LTI compensators of order 3 can be applied at each channel, so as to place $n$ eigenvalues each close to two desirable locations in the complex plane while driving the remaining $3 n$ eigenvalues arbitrarily far left in the complex plane. Specifically, consider using a compensator at each agent $i$ with transfer function $h_{i}(s)=k_{o}+\left(k_{1} s / 1+\epsilon \lambda_{f i} s\right)+\left(k_{2} s^{2} / 1+\epsilon s \lambda_{d i}+\epsilon^{2} s^{2} \lambda_{z i}\right)$, and say that we wish to place $n$ closed-loop eigenvalues at each of the roots of $s^{2}+\alpha s+\beta$. By choosing $k_{2}$ sufficiently large, $k_{1}=\alpha k_{2}$, and $k_{o}=\beta k_{2}$, and choosing $\lambda_{f i}, \lambda_{d i}$, and $\lambda_{z i}$ appropriately, $n$ closed-loop eigenvalues can be placed arbitrarily close to each root of $s^{2}+\alpha s+\beta$ as $\epsilon$ is made small, while the remaining $3 n$ eigenvalues can be moved arbitrarily far left in the complex plane (in particular, having order $1 / \epsilon$ ).

Proof: The proof is in two steps. In the first step, we show that decentralized feedback of the observation and its first two derivatives can be used to place $2 n$ closed-loop eigenvalues arbitrarily near to two locations in the complex plane, and in fact there is a parameterized family of controllers of this form that suffice. In the second step, we 
use this result to construct proper third-order LTI compensators at each channel that achieve the pole-placement specification given in the theorem statement.

Step 1: Let us study the closed-loop eigenvalues of the system when the (decentralized) control law $\mathbf{u}(t)=k_{0} \mathbf{y}(t)+k_{1} \dot{\mathbf{y}}(t)+k_{2} \ddot{\mathbf{y}}(t)$, where $k_{0}=\beta k_{2}$ and $k_{1}=\alpha k_{2}$, is used. The state-space representation of the closed loop system in this case is $\dot{\mathbf{X}}=A_{c} \mathbf{X}$, where $\mathbf{X}=\left[\begin{array}{l}\dot{\mathbf{x}} \\ \mathbf{x}\end{array}\right]$ and

$$
A_{c}=\left[\begin{array}{cc}
\left(I-k_{2} G\right)^{-1} k_{1} G & \left(I-k_{2} G\right)^{-1} k_{0} G \\
I & 0
\end{array}\right] .
$$

Using the notation $\left[\begin{array}{l}X_{1} \\ X_{2}\end{array}\right]$ for a right eigenvector of $A_{c}$, we have $A_{c}\left[\begin{array}{l}X_{1} \\ X_{2}\end{array}\right]=\lambda\left[\begin{array}{l}X_{1} \\ X_{2}\end{array}\right]$, which implies that $X_{1}=\lambda X_{2}$, and $\lambda\left(I-k_{2} G\right)^{-1} \alpha k_{2} G X_{2}+\left(I-k_{2} G\right)^{-1} \beta k_{2} G X_{2}=\lambda X_{1}$. The latter yields: $\lambda \alpha k_{2} G X_{2}+\beta k_{2} G X_{2}=\lambda^{2}\left(I-k_{2} G\right) X_{2}$, or $\left(\lambda \alpha k_{2}+\beta k_{2}+\lambda^{2} k_{2}\right) G X_{2}=\lambda^{2} X_{2}$. This means that $X_{2}$ must be an eigenvector of $G$ with, say, eigenvalue $\lambda_{i}$. In this notation, we have $\left(\lambda \alpha k_{2}+\beta k_{2}+\lambda^{2} k_{2}\right) \lambda_{i}=\lambda^{2}$, or $\lambda^{2}-\alpha k_{2} \lambda_{i} /\left(1-k_{2} \lambda_{i}\right) \lambda-\beta k_{2} \lambda_{i} /\left(1-k_{2} \lambda_{i}\right)=0$. Thus, the closed-loop eigenvalues are the roots of the characteristic equations $\lambda^{2}-\alpha k_{2} \lambda_{i} /\left(1-k_{2} \lambda_{i}\right) \lambda-\beta k_{2} \lambda_{i} /\left(1-k_{2} \lambda_{i}\right)$, for $i=1, \ldots, n$. Hence, by making $k_{2}$ sufficiently large, the coefficients of the characteristic equation can be made arbitrarily close to the coefficients of the quadratic equation $\lambda^{2}+\alpha \lambda+\beta=0$. From the continuous dependence of roots on parameters, the closed-loop poles thus come arbitrarily close to the roots of this characteristic equation (which are the two desired locations), for all $k_{2}$ sufficiently large.

Step 2: We now consider using a compensator at each channel $i$ with transfer function $h_{i}(s)=k_{o}+k_{1} s /\left(1+\epsilon \lambda_{f i} s\right)+$ $k_{2} s^{2} /\left(1+\epsilon s \lambda_{d i}+\epsilon^{2} s^{2} \lambda_{z i}\right)$, where the gains $k_{0}, k_{1}$, and $k_{2}$ are those determined in Step $1, \lambda_{f i}, \lambda_{d i}$, and $\lambda_{z i}$ are constants to be designed, and $\epsilon$ is a positive constant that will be designed sufficiently small after the other parameters have been designed. We note that this controller requires at most three memory elements at each channel to implement.

Substituting for the controllers' dynamics, one immediately finds the closed-loop characteristic polynomial. In particular, the closed-loop system's poles are the locations $s$ in the complex plane such that $Q(s)=\left(I+\epsilon s \Lambda_{f}\right)\left(I-k_{2} G+\epsilon s \Lambda_{d}+\epsilon^{2} s^{2} \Lambda_{z}\right) s^{2}-\left(I+\epsilon s \Lambda_{d}+\right.$ $\left.\epsilon^{2} s^{2} \Lambda_{z}\right) k_{1} G s-\left(I+\epsilon s \Lambda_{f}\right)\left(I+\epsilon s \Lambda_{d}+\epsilon^{2} s^{2} \Lambda_{z}\right) k_{0} G$ loses rank, where $\Lambda_{f}, \Lambda_{d}$, and $\Lambda_{z}$ are diagonal matrices with $i$ th diagonal entry given by $\lambda_{f i}, \lambda_{d i}$, and $\lambda_{z i}$, respectively. We notice that the closed-loop system has $5 n$ poles (counting multiplicities).

To continue, we note that $Q(s)$ can be written as $Q(s)=s^{2} I-$ $k_{0} G-s k_{1} G-s^{2} k_{2} G+\epsilon M_{1}(s)+\epsilon^{2} M_{2}(s)$, where $M_{1}(s)$ and $M_{2}(s)$ do not depend on $\epsilon$. Let us first consider the $2 n$ values $s$ for which $s^{2} I-k_{0} G-s k_{1} G-s^{2} k_{2} G$ loses rank. We note that these are precisely the closed-loop poles when the derivative-based controller is used, and so these values of $s$ are in two groups of $n$, arbitrarily near to the two desired pole locations. It follows easily from perturbation arguments that, thus, $n$ poles of the closed-loop system upon lead-compensator control (values $s$ such that $Q(s)$ loses rank) are arbitrarily close to each desired pole location.

What remains to be shown is that the remaining poles are order $1 / \epsilon$ and indeed can be placed in the left-half-plane. To see this, let us rewrite the Laplace-domain expression in terms of $\bar{s}=\epsilon s$. Doing so, we recover that $R(\bar{s})=\epsilon^{2} Q(\bar{s})=\bar{s}^{2}\left(I+\Lambda_{f} \bar{s}\right)\left(I-k_{2} G+\bar{s} \Lambda_{d}+\right.$ $\left.\bar{s}^{2} \Lambda_{z}\right)+\epsilon N_{1}(\bar{s})+\epsilon^{2} N_{2}(\bar{s})$. To characterize the values $\bar{s}$ such that $R(\bar{s})$ and hence $Q(\bar{s})$ lose rank, let us first consider $T(\bar{s})=\bar{s}^{2}(I+$ $\left.\Lambda_{f} \bar{s}\right)\left(I-k_{2} G+\bar{s} \Lambda_{d}+\bar{s}^{2} \Lambda_{z}\right)$. We recognize that $T(\bar{s})$ loses rank at $\bar{s}=0$ with multiplicity $2 n$, as well as at the $3 n$ values $\bar{s}$ such that $\left(I+\Lambda_{f} \bar{s}\right)\left(I-k_{2} G+\bar{s} \Lambda_{d}+\bar{s}^{2} \Lambda_{z}\right)$ loses rank. These $3 n$ values are non-zero as long as $I-k_{2} G$ is made full rank (which we shall shortly guarantee), and we choose $\Lambda_{f}, \Lambda_{d}$, and $\Lambda_{z}$ full rank and $k_{2} \neq 0$, as we shall do. In this case, we see immediately from perturbation arguments that the polynomial $R(\bar{s})$ loses rank at $2 n$ values $\bar{s}$ that approach the origin as $\epsilon$ is made small, as well as at $3 n$ other values $\bar{s}$ that approach the $3 n$ non-zero points in the complex plane where $\left(I+\Lambda_{f} \bar{s}\right)\left(I-k_{2} G+\bar{s} \Lambda_{d}+\bar{s}^{2} \Lambda_{z}\right)$ loses rank, as $\epsilon$ is made small. Rewriting all these values in terms of $s$ rather than $\bar{s}$, we see that the closed-loop system has $2 n$ poles that are close to the origin in that they do not grow as fast as $\theta(1 / \epsilon)$ (and which we have already characterized to be close to two desired locations in the complex plane), as well as $3 n$ poles of order $1 / \epsilon$ if the poles of $\left(I+\Lambda_{f}\right)\left(I-k_{2} G+\bar{s} \Lambda_{d}+\bar{s}^{2} \Lambda_{z}\right)$ are nonzero (as we will show shortly).

Finally, let us construct the controller so that the values $\bar{s}$ for which $\left(I+\Lambda_{f} \bar{s}\right)\left(I-k_{2} G+\bar{s} \Lambda_{d}+\bar{s}^{2} \Lambda_{z}\right)$ loses rank are all in the OLHP, and hence complete the proof. Clearly, $n$ of these values are the $\bar{s}$ such that $\left(I+\Lambda_{f} \bar{s}\right)$ loses rank. We can make these values negative and real by choosing each $\lambda_{f i}$, and hence $\Lambda_{F}$, positive and real.

Next, let us consider the $2 n$ values $\bar{s}$ such that $\left(I-k_{2} G+\bar{s} \Lambda_{d}+\right.$ $\bar{s}^{2} \Lambda_{z}$ ) loses rank. Since we have assumed $\Lambda_{z}$ is full rank, we can equivalently find $\bar{s}$ such that $\bar{s}^{2} I+\Lambda_{z}^{-1} \Lambda_{d} \bar{s}+\Lambda_{z}^{-1}\left(I-k_{2} G\right)$ loses rank. To continue, we note that all principal minors of $I-k_{2} G$ are full rank for all $k_{2}$ except those in a particular finite set, i.e., for all $k_{2}$ except those that are inverses of eigenvalues of the principal minors of $G$. Hence, for any design with $k_{2}$ large enough, $I-k_{2} G$ has a nested sequence of principal minors of full rank. Using any such design, $\Lambda_{z}$ can be chosen to place the eigenvalues of $\Lambda_{z}^{-1}\left(I-k_{2} G\right)$ at positive real values, according to the classical result of Fisher and Fuller (quoted as Theorem 1 above). Finally, let us choose $\Lambda_{d}=\Lambda_{z}$. In this case, we see from simple eigenanalysis that the values $\bar{s}$ for which rank is lost are the solutions of the $n$ scalar equations $\bar{s}^{2}+\bar{s}+\lambda_{i}$, where each $\lambda_{i}$ is an eigenvalue of $\Lambda_{z}^{-1}\left(I-k_{2} G\right)$. Thus, we obtain that all solutions $\bar{s}$ are in the OLHP by a proper choice of the controller parameters.

A multi-lead-compensator decentralized controller has thus been designed for an arbitrary double-integrator network, which achieves stabilization as well as a certain group pole placement. Let us stress that this group pole-placement capability gives us wide freedom to shape the dynamical response (in terms of settling and robustness properties), including by guaranteeing phase margin in the design through an inverse optimality argument (see [4]).

Let us now summarize some conceptualizations/extensions of the design, omitting details to save space.

1) Our result can be generalized to the case where each agent may make multiple observations, by noting the flexibility in combining observations in achieving stabilization [6].

2) The design that we have presented can be interpreted as comprising an estimator and a state-feedback controller. Specifically, if the pure derivative controller is used (with $k_{2}$ large), the agents can be viewed as immediately obtaining their local state (in particular, by rearranging their initial conditions in such a way that the second-derivative estimate and hence local state estimate are precise); thus, state feedback control can be used. In practice, an immediate estimation of initial conditions is not implementable. Instead, the lead compensator design achieves estimation at a faster time scale than the state feedback response, but not immediately. We stress that, although such fast observers are widely used in centralized control, our methodology is fundamentally different from the centralized case in that the state estimation is only possible when the feedback is in force-that is, the estimation and control tasks are not decoupled.

3) In autonomous-agent networks, robustness to agent failure is an important concern. Agent failure can, for instance, be modeled as certain agents being unmeasurable by all other agents after a 
certain time. The robustness question is whether the rest of the agents can still achieve stability using the original controller design. Mathematically, this is the problem of whether, if all rows and columns in the sensing structure associated with the failed agents are removed, stability in the reduced dimensional system remains. We notice that current literature on decentralized controller design does not address this important issue. For instance, the dominant channel design in [18] is highly sensitive to the failure of the dominant channel, due to the significant role played by this single channel in stabilization. In the contrast, since in our lead-compensator design, all agents contribute roughly equally to the stabilization task, this design appears to be more robust to agent failure. For broad classes of sensing structures, e.g., those for which $I-k_{2} G$ is strictly $D$-stable through a diagonal (signpattern) scaling, stability is maintained in the presence of any number of agent failures. This is because the eigenvalues of the principal submatrices of $\Lambda_{z}^{-1}\left(I-k_{2} G\right)$ remain in the OLHP and hence so do those of the closed-loop state matrix. Clearly, subclasses of $G$ that satisfy the above include strictly D-stable, positive definite, and grounded Laplacian topology matrices (see Fig. 1 for a full illustration).

4) We stress that, for arbitrary plants with no decentralized fixed modes, exact pole assignment has been achieved in the literature, [19]. In comparison, we are here only achieving an approximate pole assignment, and for a very specific class of autonomousagent networks. However, the philosophy of our design differs drastically from the classical pole-assignment design, in that the agents share observation/actuation effort and complexity; in contrast, in the classical methods, the dynamics are made controllable and observable from a single channel through perturbation, and the effort/complexity are concentrated at this single channel. Our concurrent work [4] has made the benefit of the new scheme explicit in specific examples (for example, an orders-of-magnitude advantage in actuation are realized in a cycle-graph example), and given a detailed conceptualization of the new scheme. We also note that numerous studies including ours demonstrate the difficulties that arise in using a single dominant channel [18].

5) Our design methodology is based on a high-gain or time-scale-assignment philosophy. This time-scale assignment (or high gain) approach is in analogy with the classical designs used for centralized plants, and in its essence is needed for stabilization and high-performance control. The time-scale assignment design (like all controller designs) must be tuned/refined with several performance metrics in mind, including disturbance- and noiseresponse metrics and robustness measures. We leave such further refinement of the design to future work. We note that the design under actuator saturation described below gives a parametrized family of controllers, which we expect will facilitate design to trade off settling and noise-sensitivity properties.

6) The careful reader will note that the gain $k_{2}$ can be chosen to be positive or negative. Either choice permits completion of the control task, but the choice does impact the values of other controller parameters (gains, controller pole locations) needed for stabilization and group pole placement.

7) Although the slow $(\theta(1))$ poles - whose locations can be designed through our approach - dominate the settling behavior of the dynamics, refining the locations of the fast poles may benefit other aspects of the network's response, e.g., sensitivity to sensor noise and actuation required over a short time horizon. We stress the Fisher and Fuller's work [23] provides a placement of the fast poles in the OLHP for general graph topologies. Meanwhile, for particular matrix classes, numerous tools are available for placing eigenvalues through scaling, see [24] and references contained therein.

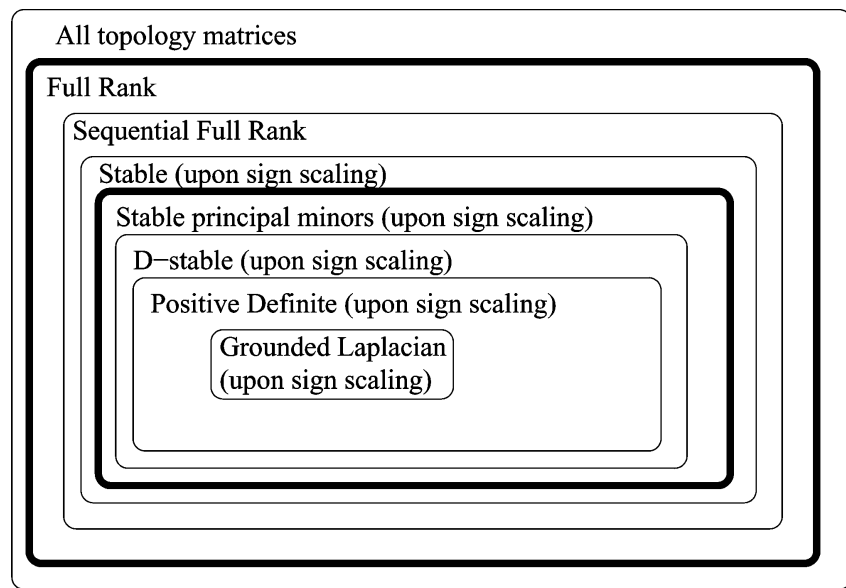

Fig. 1. We diagram several matrix classes that are of interest in representing a double integrator network's sensing topology. A multi-lead-compensator design is possible whenever the topology matrix is full rank, and a design that is robust to agent failures is possible if the topology matrix has stable principal minors to within a sign scaling (a scaling of each row by \pm 1 ).

\section{Stabilization Under Actuator SATURATion}

We show that a multi-lead-compensator can semi-globally stabilize a double-integrator network under saturation, for an arbitrary topology matrix $G$. We stress again that the double-integrator network has $2 n$ poles at the origin. Hence, the result is an expansion of the sufficient condition for the existence of a stabilizing controller provided in [30].

In the following Theorem 3, we show the design of the stabilizing multi-lead-compensator using a low-gain strategy. More specifically, a scaling of the compensator design presented in Theorem 2 is proposed, that yields semi-global stabilization under actuator saturation. Here is the result:

Theorem 3: Consider a double-integrator network with input saturation, and arbitrary invertible topology matrix $G$. Say that compensators $h_{i}(s)=k_{o}+k_{1} s /\left(1+\epsilon \lambda_{f i} s\right)+k_{2} s^{2} /\left(1+\epsilon s \lambda_{d i}+\epsilon^{2} s^{2} \lambda_{z i}\right)$ have been designed for each agent $i$ according to Theorem 1 , so that stabilization is achieved in the double-integrator network without saturation. Then the network with input saturation can be semiglobally stabilized using at each agent $i$ the parameterized family of proper LTI compensators $\hat{h}_{i, \hat{\epsilon}}(s)=k_{o} \hat{\epsilon}^{2}+$ $\hat{\epsilon} k_{1} s /\left(1+(\epsilon / \hat{\epsilon}) \lambda_{f i} s\right)+k_{2} s^{2} /\left(1+(\epsilon / \hat{\epsilon}) s \lambda_{d i}+\left(\epsilon^{2} / \hat{\epsilon}^{2}\right) s^{2} \lambda_{z i}\right)$. That is, for any specified ball of plant and compensator initial conditions $W$, there exists $\hat{\epsilon}^{*}(W)$ such that, for all $0<\hat{\epsilon} \leq \hat{\epsilon}^{*}(W)$, the compensator with the transfer function $\hat{h}_{i, \hat{\epsilon}}(s)$ at each channel achieves local stabilization of the origin and contains $W$ in its domain of attraction.

Proof: To prove the result, we will need to study the system (1) (i.e., study the system without saturation) in two cases: first, when the controller $H(s)=\operatorname{diag}\left(h_{i}(s)\right)$ is used, and second when the controller $\widehat{H}(s)=\operatorname{diag}\left(\hat{h}_{i}(s)\right)$, for some appropriately-chosen initial conditions.

Without loss of generality, we can limit ourselves to examining the response from the plant initial conditions, since the component of the response due to the precompensator initial conditions can be made arbitrarily small through static pre- and post-scaling of the compensator at each agent, see e.g., [31].

In order to prove that the proposed controller semiglobally stabilizes the double-integrator network under input saturation, it suffices to show that, for any bounded set of initial conditions $W$ the $\infty$-norm of the input $\|\mathbf{u}(t)\|$ where $t \geq 0$ remains upper bounded by 1 , and also the dynamics without saturation are asymptotically stable. Thus, we can verify semi-global stabilization by showing that, for any bounded set 
of initial conditions $W$ the norm of the input $\|\mathbf{u}(t)\|$ where $t \geq 0$ scales by $\hat{\epsilon}$ and further the closed-loop dynamics without actuator saturation are asymptotically stable. Let us prove this through a spectral argument. Specifically, let us relate the response when the new scaled controller is used to response of the unscaled controller, and use this relationship to prove stability.

Let us first consider applying the new scaled controller, i.e., the controller with transfer function $\widehat{H}(s)$. In the Laplace domain, the closedloop dynamics of the double-integrator network ignoring saturation (when this scaled controller is used) are given by

$$
\begin{aligned}
& s^{2} \mathbf{X}(s)-s \mathbf{x}(0)-\dot{\mathbf{x}}(0) \\
& \quad=k_{o} \hat{\epsilon}^{2} G \mathbf{X}(s)+\left(I+\frac{\epsilon}{\hat{\epsilon}} \Lambda_{f} s\right)^{-1} \hat{\epsilon} s k_{1} G \mathbf{X}(s) \\
& \quad+\left(1+\frac{\epsilon}{\hat{\epsilon}} \Lambda_{d} s+\frac{\epsilon^{2}}{\hat{\epsilon}^{2}} \Lambda_{z} s^{2}\right)^{-1} k_{2} s^{2} G \mathbf{X}(s)
\end{aligned}
$$

where $\Lambda_{f}, \Lambda_{d}$, and $\Lambda_{z}$ are diagonal matrices whose $i$ th diagonal entries are $\lambda_{f i}, \lambda_{d i}$, and $\lambda_{z i}$, respectively.

Let us apply the change of variables $\hat{\epsilon} \bar{s}=s$, and scale both sides of (3) by $1 / \widehat{\epsilon}^{2}$. The closed-loop system dynamics in terms of $\bar{s}$ in the Laplace domain becomes

$$
\begin{aligned}
& \bar{s}^{2} \mathbf{X}(\hat{\epsilon} \bar{s})-\frac{1}{\hat{\epsilon}} \bar{s} \mathbf{X}(0)-\frac{1}{\hat{\epsilon}^{2}} \dot{\mathbf{x}}(0) \\
& \quad=k_{o} G \mathbf{X}(\hat{\epsilon} \bar{s})+\left(I+\epsilon \Lambda_{f} \bar{s}\right)^{-1} \bar{s} k_{1} G \mathbf{X}(\hat{\epsilon} \bar{s}) \\
& \quad+\left(I+\epsilon \Lambda_{d} \bar{s}+\epsilon^{2} \Lambda_{z} \bar{s}^{2}\right)^{-1} k_{2} \bar{s}^{2} G \mathbf{X}(\hat{\epsilon} \bar{s})
\end{aligned}
$$

From (4), we get

$$
\begin{aligned}
\mathbf{X}(\hat{\epsilon} \bar{s}) & =M \frac{1}{\hat{\epsilon}} \bar{s} \mathbf{x}(0)+M \frac{1}{\hat{\epsilon}^{2}} \dot{\mathbf{x}}(0) \\
& =\frac{1}{\hat{\epsilon}^{2}}(M \hat{\epsilon} \bar{s} \mathbf{x}(0)+M \dot{\mathbf{x}}(0))
\end{aligned}
$$

where $M=\left(\bar{s}^{2} I-k_{o} G-\left(I+\epsilon \Lambda_{f} \bar{s}\right)^{-1} \bar{s} k_{1} G-\left(1+\epsilon \Lambda_{d} \bar{s}+\right.\right.$ $\left.\left.\epsilon^{2} \Lambda_{z} \bar{s}^{2}\right)^{-1} k_{2} \bar{s}^{2} G\right)^{-1}$.

On the other hand, we note that using the original controller design with transfer function $H(s)$ and initial conditions $(\hat{\epsilon} \mathbf{x}(0), \dot{\mathbf{x}}(0))$ (and calling the state $X()$ as in our previous development), the closed loop dynamics for the double integrator network ignoring saturation becomes (in terms of $\bar{s}$, where $\epsilon s=\bar{s}$ )

$$
\overline{\mathbf{X}}(\bar{s})=M \hat{\epsilon} \bar{s} \mathbf{x}(0)+M \dot{\mathbf{x}}(0)
$$

Here, we have used the notation $\overline{\mathbf{X}}(\bar{s})$ for the Laplace transform of the state to avoid confusion.

Equations (5) and (6) together inform us that $\hat{\epsilon} \mathbf{X}(\hat{\epsilon} \bar{s})$ is equal to $\overline{\mathbf{X}}(\bar{s})$ scaled by $1 / \hat{\epsilon}$, assuming the initial conditions for the two systems are chosen commensurately. That is, the double-integrator network's response upon use of the controller $\hat{H}_{\hat{\epsilon}}(s)$ (with initial condition $\mathbf{x}(0)$ and $\dot{\mathbf{x}}(0)$ ) has the same shape as the response of the double-integrator network upon use of the controller with transfer function $H(s)$ (with the initial conditions $(\hat{\epsilon} \mathbf{x}(0), \dot{\mathbf{x}}(0)))$, but scaled in amplitude by $1 / \hat{\epsilon}$ and in frequency also by $1 / \hat{\epsilon}$. Using this scaling and considering the inputs (see the right sides of (3) and (5), we immediately see that the input upon use of the scaled controller has the same shape as the input upon use of the original controller (assuming commensurate initial conditions), but scaled in amplitude by $\hat{\epsilon}$ and in frequency by $1 / \hat{\epsilon}$.

Now let us use this relationship to show that, for any closed and bounded set of initial conditions $\mathcal{W}$, the parameter $\hat{\epsilon}$ can be chosen so that 1 ) actuator saturation is not activated and so the linear model is in force and 2) the closed-loop linear model remains stable, thus guaranteeing that the set is in the domain of attraction. To do so, let us consider applying the scaled controller with some small $\hat{\epsilon}$ (specifically, $\hat{\epsilon} \ll 1$ ) when the initial condition is in $\mathcal{W}$. We notice that these responses are scaled versions of the responses when the original controller is used, for another bounded set of initial conditions (which is a subset $\mathcal{W}$ since $\hat{\epsilon}$ is assumed less than 1). Noting that the original controller achieves stability, we know that the maximum infinity-norm of the input is bounded over the initial conditions in the bounded set. Recalling that the amplitude of the input scales by $\hat{\epsilon}$ when the scaled controller is used, we see that by choosing $\hat{\epsilon}$ sufficiently small we can avoid saturation for all initial conditions in the ball $\mathcal{W}$. Furthermore, we directly recover from (5) and (6) that the closed-loop poles scale with $\hat{\epsilon}$ upon use of the scaled controller, and so asymptotic stability is maintained. Thus, semi-global stabilization is achieved.

In the above theorem, we have shown that a low-gain scaling of a (decentralized) multi-lead-compensator stabilizes a double-integrator network under saturation, for an arbitrary full-rank topology matrix $G$. Thus, we have fully addressed design of low-gain decentralized controllers for the double-integrator network with saturation. Let us conclude with three remarks about the design:

1) Let us distinguish our approach with the traditional low-gain approach for centralized systems with saturation [32], [33]. For centralized plants, actuation capabilities are subdivided between the observer and state feedback. In contrast, the double-integrator network requires integrated design of the entire dynamical controller, and hence we need a scaling of the full design to address control under saturation.

2) It is an open question as to whether decentralized plants with repeated $j \omega$-axis eigenvalues (and with all CLHP eigenvalues and all decentralized fixed modes in the OLHP) can be semi-globally stabilized. This first result shows that there is some promise for achieving semi-global stabilization broadly.

3) With some effort, the scaling used to achieve stabilization under saturation can also be shown to permit stabilization under arbitrary observation delay, and to provide robustness to observation delay. Alternately, a delay-based implementation of the multiple-derivative scheme naturally permits control under intrinsic observation delay. We expect to pursue this direction in future work.

\section{EXAMPLE}

We include an example to illustrate the design methodology. In particular, let us consider a double-integrator network with topology matrix $G=\left[\begin{array}{cccc}0 & 1 & 0 & 0 \\ 0 & 0 & 1 & 0 \\ 0 & 0 & 0 & 1 \\ 1 & 0 & 1 & 0\end{array}\right]$. The double-integrator network with this topology matrix is particularly difficult to control, because the agents do not have any information about their own state, and control instead critically depends on using a cycle of observations. It is easy to check that proportional-derivative controllers, as typically considered in the autonomous-agent network control literature, cannot achieve stabilization. In fact, one can verify that the agents' controllers must in total have eight zeros (and at least eight poles). We notice our design divides this required controller complexity among the agents. Applying the design procedure, we find that a decentralized controller of the form given in Theorem 2 with parameters $k_{0}=-5, k_{1}=-10, k_{2}=-5$, $\Lambda_{f}=I, \Lambda_{d}=\Lambda_{z}=\operatorname{diag}(200,1,-50,1)$, and $\epsilon=0.005$ achieves stabilization and in fact makes the real parts of all closed-loop poles less than -0.7 . More specifically, the design induces two time scales, with slow poles roughly near $s=-1$ and fast poles of magnitude approximately 200 . The presented design has three stable controllers and one unstable one; it is easy to check the larger corner frequency of each 
agent's controller is approximately $200 \mathrm{~Hz}$, giving an indication of the noise sensitivity of the design. We note that the design achieves similar magnitude gains/actuations in each channel as well as moderate noise sensitivity compared to single-dominant-channel-type designs, giving an indication of the benefits of our approach. We note that the design can be scaled to achieve stabilization under saturation (and concurrently lower sensitivity to noise), at the cost of slower convergence.

We kindly ask the reader to see [4] for other examples/simulations of multiple-derivative-based decentralized controllers, including one comparing this design (in terms of complexity and actuation) with the designs that achieve stabilization by making a single channel controllable and observable.

\section{CONCLUSION}

We have proposed a simple decentralized controller architecture for double-integrator networks. We show that the lead-compensator implementation of the derivative controller up to the order of two can stabilize the plant and realize pole placement, even in the presence of actuator saturation. The new controller architecture has advantages over traditional controller design in terms of disturbance rejection and robustness to agent failures.

\section{REFERENCES}

[1] Y. Wan and S. Roy, "A scalable methodology for evaluating and designing coordinated air traffic flow management strategies under uncertainty," IEEE Trans. Intell. Transport. Syst., vol. 9, pp. 644-656, Aug. 2008.

[2] Y. Wan, S. Roy, and A. Saberi, "Designing spatially-heterogeneous strategies for control of virus spread," IET Syst. Biol., vol. 2, no. 4, pp. 184-201, 2008.

[3] Y. Wan, S. Roy, A. Saberi, and B. Lesieutre, "A flexible stochastic automaton-based algorithm for network self-partitioning," Int. J. Distrib. Sensor Networks, vol. 4, no. 3, pp. 223-246, 2008.

[4] Y. Wan, S. Roy, A. Saberi, and A. Stoorvogel, "A multiple-derivative and multiple-delay paradigm for decentralized controller design: Introduction using the canonical double-integrator network," in Proc. AIAA Guid., Navig., Control Conf., Honolulu, HI, Aug. 18-21, 2008, [CD ROM].

[5] Y. Wan, S. Roy, and A. Saberi, "A new focus in the science of networks: Toward methods for design," in Proc. Royal Soc. A, Mar. 2008, vol. 464, pp. 513-535.

[6] S. Roy, A. Saberi, and K. Herlugson, "Formation and alignment of distributed sensing agents with double-integrator dynamics," IEEE Press Monograph Sensor Network Oper., pp. 126-157, May. 2006.

[7] C. W. Wu and L. Chua, "Application of kronecker products to the analysis of systems with uniform linear coupling," IEEE Trans. Circuits Syst. I, vol. 42, no. 10, pp. 775-779, Oct. 1995.

[8] C. W. Wu and L. Chua, "Application of graph theory to the synchronization in an array of coupled nonlinear oscillators," IEEE Trans. Circuits Syst. I, vol. 42, no. 8, pp. 494-497, Aug. 1995.

[9] S. H. Strogatz and I. Stewart, "Coupled oscillators and biological synchronization," Sci. Amer., vol. 269, no. 6, pp. 102-109, Dec. 1993.

[10] J. A. Fax and R. M. Murray, "Information flow and cooperative control of vehicle formations," IEEE Trans. Autom. Control, vol. 49, no. 9, pp. 1465-1476, Sep. 2004.

[11] A. Pogromsky and H. Nijmeijer, "Cooperative oscillatory behavior of mutually coupled dynamical systems," IEEE Trans. Circuits Syst. I, vol. 48, no. 2, pp. 152-162, Feb. 2001.

[12] J. Baillieul and P. J. Antsaklis, "Control and communication challenges in networked real-time systems," Proc. IEEE, vol. 95, no. 1, pp. 9-28, Jan. 2007.

[13] S. Roy, A. Saberi, and A. Stoorvogel, "Toward a control theory for networks," Int.. J. Robust Nonlin. Control, vol. 17, no. 10-11, pp. 897-897, Jul. 2007.

[14] Z. Duan, J. Wang, G. Chen, and L. Huang, "Stability analysis and decentralized control of a class of complex dynamical networks," Automatica, vol. 44, pp. 1028-1035, 2008.
[15] W. Ren, "On consensus algorithms for double-integrator dynamics," IEEE Trans. Autom. Control, vol. 53, no. 7, pp. 1503-1509, Jul. 2008.

[16] S. Wang and E. J. Davison, "On the stabilization of decentralized control systems," IEEE Trans. Autom. Control, vol. AC-18, pp. 473-478, Oct. 1973.

[17] D. Siljak, Decentralized Control of Complex Systems. Boston, MA: Academic Press, 1994.

[18] J. P. Corfmat and A. S. Morse, "Decentralized control of linear multivariable systems," Automatica, vol. 12, pp. 479-495, 1976.

[19] E. J. Davison and T. N. Chang, "Decentralized stabilization and pole assignment for general proper systems," IEEE Trans. Autom. Control, vol. AC-35, no. 6, pp. 652-664, Jun. 1990.

[20] Y. Wan, S. Roy, A. Saberi, and A. Stoorvogel, "A multiple-derivative and multiple-delay paradigm for decentralized controller design: Uniform rank systems," Dyn. Continuous, Discrete, Impulsive Syst., Special Issue in Honor of Dr. Hassan Khalil's 60th Birthday, to be published.

[21] S. Roy, Y. Wan, and A. Saberi, "A network control theory approach to virus-spread mitigation," in Proc. IEEE Homeland Security Conf., Boston, MA, May 2009, pp. 599-606.

[22] S. Boyd, "Convex optimization of graph laplacian eigenvalues," in Proc. Int. Congress Math., 2006, vol. 3, pp. 1311-1319.

[23] M. E. Fisher and A. T. Fuller, "On the stability of matrices and the convergence of linear iterative processes," in Proc. Cambridge Philosoph. Soc., 1958, vol. 45, pp. 417-425.

[24] S. Roy, A. Saberi, and P. Petite, "Scaling: A canonical design problem for networks," in Proc. 50th Amer. Control Conf., Minneapolis, MN, Jun. 2006, vol. 80, pp. 1342-1353.

[25] Y. Wan, S. Roy, X. Wang, A. Saberi, T. Yang, M. Xue, and B. Malek, "On the structure of graph edge designs that optimize the algebraic connectivity," in Proc. 47th IEEE Conf. Decision Control, Cancun, Mexico, Dec. 9-11, 2008, pp. 805-810.

[26] S. Roy, Y. Wan, and A. Saberi, "On time-scale designs for networks," Int. J. Control, vol. 82, no. 7, pp. 1313-1325, Jul. 2009.

[27] B. D. O. Anderson and J. B. Moore, "Time-varying feedback laws for decentralized control," IEEE Trans. Autom. Control, vol. AC-26, no. 10, pp. 1133-1139, Oct. 1981.

[28] A. Saberi, B. M. Chen, and P. Sannuti, Loop Transfer Recovery: Analysis and Design. New York: Springer-Verlag, 1993.

[29] D. A. O'Conner and T. J. Tarn, "On stabilization by state feedback for neutral differential and difference equations," IEEE Trans. Autom. Control, vol. AC-28, no. 5, pp. 615-619, May 1983.

[30] A. Stoorvogel, A. Saberi, C. Deliu, and P. Sannuti, "Decentralized stabilization of time-invariant systems subject to actuator saturation," in Advanced Strategies in Control Systems With Input and Output Constraints, ser. LNCIS, S. Tarbouriech, Ed. et al. New York: SpringerVerlag, 2006.

[31] S. Roy, Y. Wan, A. Saberi, and B. Malek, "An alternative approach to designing stabilizing compensators for saturating linear time-invariant plants," Int. J. Robust Nonlin. Control, vol. 20, pp. 1520-1528, Nov. 2010.

[32] Z. Lin and A. Saberi, "Semi-global exponential stabilization on linear systems subject to input saturation via linear feedbacks," Syst. Control Lett., vol. 21, pp. 225-239, 1993.

[33] Z. Lin and A. Saberi, "A semi-global low-and-high gain design technique for linear systems with input saturation-Stabilization and disturbance rejection," Int. J. Robust Nonlin. Control, vol. 5, pp. 381-398, 1995. 